Communications in Physics, Vol. 28, No. 3 (2018), pp. 201-216

DOI:10.15625/0868-3166/28/3/12670

\title{
TUNING ELECTRONIC TRANSPORT PROPERTIES OF ZIGZAG GRAPHENE NANORIBBONS WITH STONE-WALES DEFECT
}

\author{
NGUYEN THANH TIEN ${ }^{a}$, , BUI THAI HOC ${ }^{a}$, NGUYEN VAN UT ${ }^{a}$ AND LE TUAN $^{b}$ \\ ${ }^{a}$ College of Natural Sciences, Can Tho University \\ 3-2 Road, Can Tho City, Vietnam \\ ${ }^{b}$ School of Engineering Physics, Hanoi University of Science and Technology \\ 1 Dai Co Viet Road, Hanoi, Vietnam \\ ${ }^{\dagger} E$-mail: nttien@ctu.edu.vn
}

Received 13 June 2018

Accepted for publication 14 August 2018

Published 31 August 2018

\begin{abstract}
Influences of the symmetric Stone-Wales (SW) defect on the electronic transport properties of the zigzag graphene nanoribbons (ZGNRs) have been studied using ab initio simulation based on the density functional theory (DFT) combined with non-equilibrium Green's function $(N E G F)$ technique. The calculated transmission spectra $T(E)$ at various bias windows, device densities of states (DDOS), current characteristics as well as local density of states (LDOS) of the defective asymmetric and symmetric ZGNRs are presented in comparison with those for the pristine ZGNRs. The metallic character of the electronic transport in both asymmetric and symmetric ZGNRs has been established: the current has a semiconductor behavior, with negative differential resistance (NDR) effect. Symmetric SW defect, as a most unfavorable SW defect type for electric conductance, remarkably reduces the current values, but does not change the character of conductivity in both the asymmetric and symmetric ZGNRs. NDR has been explained by the SW defect-induced alteration of the number of frontier molecular orbitals entering bias windows.
\end{abstract}

Keywords: density-functional theory, non-equilibrium, Green function, electronic transport, graphene nanoribbon, transmission spectrum, current-voltage characteristics.

Classification numbers: 31.15.A-; 71.15.Mb; 73.22.Pr.

(C)2018 Vietnam Academy of Science and Technology 


\section{INTRODUCTION}

Discovery of graphene by Novoselov and Geim [1,2], as a two-dimentional $s p^{2}$-hybridized high crystalline material with electric conductivity controllable by adjusting narrow bandgap near to Dirac point, has ignited an enormous interest of researchers. Opening bandgap graphene nanoribbons (GNR) with zigzag or armchair edges are the most promising building material in future nanoelectronic devices, for instance, in GNRFETs [3,4]. A list of reasons, like the modulation of graphene or GNR properties at nanoscale by using the general method of doping or chemical functionalization, as well as a choice of substrates where graphene is grown from, plus the impossibility to work with absolutely defect-free material, can lead to variety of defects [5-7]. In comparison of the case for bulk crystals, the investigation of defects in two-dimentional crystalline materials has been less abundant for a long time until the large-scale graphene availability.

Among the reported defects the 5eV-high formation energy Stone-Wales (SW) defect transforms four hexagons into two heptagons and two pentagons by rotating one C-C bond by $90^{\circ}$, but retains the same number of atoms like in perfect graphene, without dangling bonds [8]. Weaker bonds around defects cause an increase of thermal conductivity, a worse durability and may dramatically alter electronic and quantum-transport properties of graphene/GNRs. Lahiri [6] showed the possibility of using ribbons of graphene with defect as a metallic wire for interconnection or element of device structure. Li et. al. in his systematic study [8-11] has indicated the different character of electric transport in asymmetric and symmetric ZGNRs, where asymmetric ZGNRs behave as a conventional conductor. Liu [12] has mentioned about negative differential resistance (NDR) in zigzag graphene/graphene nanoribbon structures. Moreover, Ren [13] has emphasized that SW defect influence is more obvious for symmetric ZGNR and there NDR can take place.

In this work, based on first-principles calculation, we have studied a particular SW defect formation in order to highlight the influences on the electronic transport properties in ZGNRs in the 0-1.8 V bias range, correspondingly referenced to the perfect ZGNRs of the same widthnesses. The work is organized as the following: firstly there is a description about a single SW defect in the relaxed defective ZGNRs, then transmission spectra $T(E)$ and device density of state (DDOS) at various bias values, local density of state (LDOS), 3-D contours of current from bias and electron energy are compared between the defective and pristine ZGNRs for highlighting the difference of quantum transport in these structures.

\section{SIMULATION MODELS AND CALCULATION METHODS}

The atomically resolved GNR reconstruction has been performed by using the density functional theory (DFT) utilized in the first-principles computational package Atomistix toolkit (ATK). We use the generalized gradient approximation (GGA) formulated by Perdew and Burke and Ernzerhof (PBE) [14] to describe the exchange and correlation. All geometries were converged until the forces acting on all atoms are no less than $0.01 \mathrm{eV} / \AA$, and the calculations are performed at $\mathrm{T}$ $=300 \mathrm{~K}$. The Brillouin zone was deviced by $1 \times 1 \times 100 \mathrm{k}$-point mesh based on the MonkhorstPack scheme [15]. The electrostatic potentials were determined on a real-space grid with a mesh cutoff energy of $150 \mathrm{Ry}$ and double-zeta-double-polarized basis sets of local numerical orbitals were employed for all atoms. Van der Waals interactions were accounted for by using Grimme's DFTD2 empirical dispersion correction to the PBE [16]. 
(a)
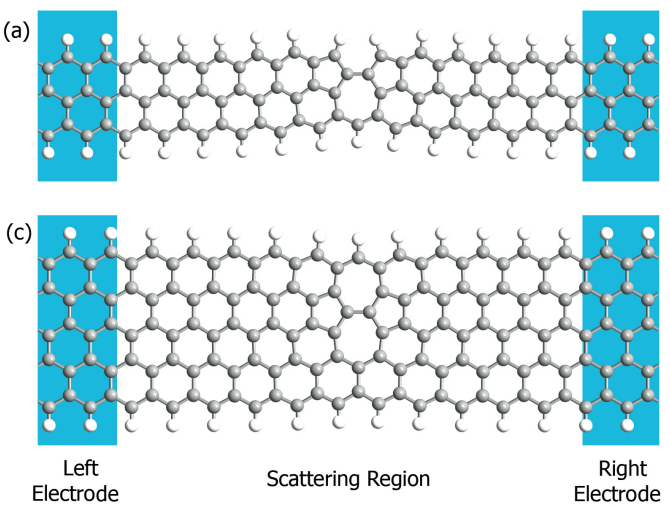

(b)

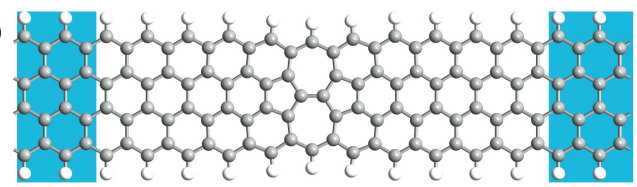

(d)

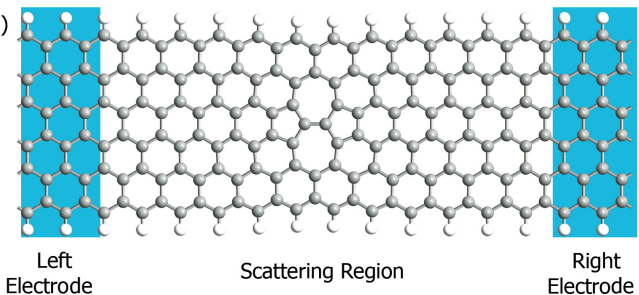

Fig. 1. Schematics of the reconstructed ZGNRs with $\mathrm{SW}$ defect: a) 55-7 SW N=3, b) 55-77 SW N=4, c) 55-77 SW N=5, d) 55-77 SW N=6.

Using the optimized structures, we constructed device geometries (see Fig. 1), which consists of the left and the right regions and the central (scattering) region (i.e., a two probe configuration). The electrodes are modeled as an electron gas with a fixed chemical potential. The transmission is calculated along the z-direction. Quantum transport simulations are implemented by using the non-equilibrium Green's function (NEGF) technique. The morphological model is built in such a way that the central region contains an optimized nanostructure with the SW defect, which is surrounded by two electrodes consisting of one unit cell on each side. In the quantum transport simulation, the transmission spectra, $T(E)$, as a function of electron energy, $E$, and the $I-V$ characteristics are calculated by the Laudauer-Büttiker formula.

The transmission function at energy $E$ and bias $V_{b}$ is calculated through the Landauer formula [17].

$$
T\left(E, V_{b}\right)=\operatorname{Tr}\left[\Gamma_{L}\left(E, V_{b}\right) G\left(E, V_{b}\right) \Gamma_{R}\left(E, V_{b}\right) G^{\dagger}\left(E, V_{b}\right)\right],
$$

where $G^{\dagger}$ and $G$ represent the retarded and advanced Green functions of the scattering region, respectively. $\Gamma_{L / R}$ are the coupling functions from the left and right leads, respectively.

The current is calculated by integrating the transmission function over the energy bias window by the following formula [18]:

$$
I\left(V_{b}\right)=\frac{2 e}{h} \int_{-\infty}^{\infty} T\left(E, V_{b}\right)\left[f\left(E-\mu_{L}\right)-f\left(E-\mu_{R}\right)\right] d E,
$$

where $f\left(E-\mu_{L / R}\right)$ are the Fermi distribution functions of the electrons in the leads; $\mu_{L}=E_{f}+$ $e V_{b} / 2$ and $\mu_{R}=E_{f}-e V_{b} / 2$ are the electrochemical potentials of the left and right leads, with $E_{F}$ is the Fermi energy at zero bias.

The equilibrium density matrix is evaluated by a contour integration method. The real space grid techniques are used with the energy cutoff of $150 \mathrm{Ry}$ as convergence criteria. The k-point sampling based on the Monkhorst-Pack scheme is $1 \times 1 \times 100$ along the $\mathrm{x}, \mathrm{y}$, and $\mathrm{z}$ axes, respectively, 
where the $\mathrm{z}$ is the electronic transport direction. In addition, the results of the electronic transport properties of the pristine configuration are also shown for comparison.

\section{RESULTS AND DISCUSSION}

In our simulation, the relaxed ZGNR structures with the SW defects are analyzed, where the number of letters 5 and 7 indicates the numbers of pentagons and heptagons, correspondingly. In this context, Fig. 1 presents the optimized structures of the systems with a SW defects surrounding by the hexagons with graphene sites in the middle of the scattering regions. In details, the relaxed atomic geometries of SW defects are shown in Fig. 2. For the 55-7 SW N=3 defect (Fig. 2a), the bond angle in the pentagon, adjacent to the heptagon, is reduced from $120^{0}$ to $112.8^{0}$, and correspondingly the bond angle in the heptagon of the asymmetric SW defect is increased to $138,22^{0}$. The first is increasing in range of $114.24^{0}-115.9^{0}$ along with $\mathrm{N}=4,5,6$ (Figs. 2 (b), (c) and (d), while the second increases to $140.27^{\circ}$. The conjunction $\mathrm{C}-\mathrm{C}$ bond of the two pentagons shrinks to $1.35 \AA$ for $\mathrm{N}=3$ and stabilized at $1.34 \AA$ for the rest cases of $\mathrm{N}$. The width of SW defects along the length axis direction is shrinking from $4.92 \AA$ to $5.61 ; 5.54 ; 5.53$ and $5.51 \AA$, or on $14.02 ; 12.60 ; 12.40$ and 12.0 percent, correspondingly, in comparison with the pristine ZGNRs with $\mathrm{N}=3,4,5,6$. No distinct change is found for the length of $\mathrm{H}-\mathrm{C}$ bond at the edge and the length bond is $1.08 \AA$. The calculated data is consistent with other works [19,20]. Therefore, the effect of symmetric SW defects on the geometry modification is limited to the defects area and this configuration still keeps the mirror reflections with respect to their axis.
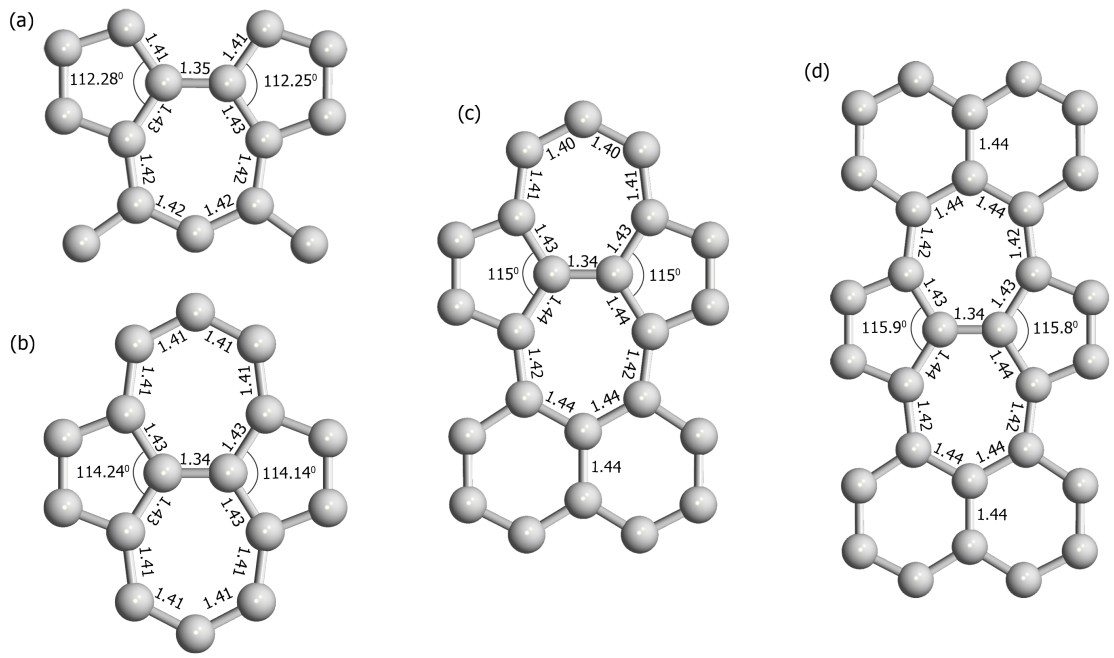

Fig. 2. Top view of SW defect in the reconstructed ZGNR nanostructures of the (a) 55-7 SW N=3; (b) 55-77 SW N=4; (c) 55-77 SW N=5; and (d) 55-77 SW N=6. The C-C bond length in $\AA$, and the angles in degree. 


\section{The 55-7 SW N=3 and 5 defects}

In order to investigate the passivation effects on transport of ZGNR, in Figs. 3a and 3c, we compare the transmission spectra $T(E)$ at zero voltage of the defective 55-7 SW N=3 and 5 with the pristine ZGNR devices. The intensity of $T(E)$ for 55-77 $\mathrm{N}=3$ case decreases significantly in most of energy levels. It becomes smaller at the Fermi level and drops sharply with decreasing electron energy away from the Fermi energy, that gives the first transmission plateau lowered to nearly zero in the range $(-1.6 \mathrm{eV},-2.2 \mathrm{eV})$, indicating the metal-semiconductor transition. More importantly, strong electron backscattering, induced by the coupling between all the states, is expanded then leads to full suppression of the conduction channel at particular resonance energies. Accordingly, a smooth conductance valley around $1.89 \mathrm{eV}$, corresponding to the complete electron backscattering, is observed. When a Stone-Wales defect in the ZGNR with $\mathrm{N}=5$, the transmission spectrum changes a lot, and one can find that the transmission steps are destroyed, instead one transmission disappears at $1.33 \mathrm{eV}$ energy sites. The transmission peak at $0 \mathrm{eV}$ has increased from one to two, indicating that the Stone-Wales defects effectively change transport properties of the defective ZGNR N=5 device.

Figs. $3 \mathrm{~b}$ and $3 \mathrm{~d}$ compare the DDOS profiles for 55-7 SW N=3 and 5 (short dash dot curves) with that of a similar system with no defect (solid curves). As depicted in these figures, each the DDOS profile also exhibits a peak about the corresponding Fermi level. These peaks correspond to the mentioned peaks observed in Figs. 3a and 3c. Comparing $T(E)$ with DDOS, it can be seen that two more peaks appear in the 55-77 SW N=5 $T(E)$, corresponding to the energies of -0.28 $\mathrm{eV}$ and $1.33 \mathrm{eV}$. More interesting is that the transmission dips of 55-77 SW N=5 appear just right in the energies of $-0.28 \mathrm{eV}$ and $1.33 \mathrm{eV}$, which is the same as that of the two more DDOS peaks that appear.

The current-voltage characteristics (short dash dot curvess) of 55-7 SW N=3 and 5 systems with that (solid curvess) of the systems, whose central scattering region contains no defects, have been shown in Figs. $4 \mathrm{a}$ and $4 \mathrm{~b}$. As observed from these figures, $I-V$ characteristics are almost linear for pristine ZGNR N=3, 5 cases proving that ZGNR $\mathrm{N}=3,5$ are metal and $I-V$ curves are consistent with Ohm's law in the range of 0-2.0V. In fact, all asymmetric ZGNRs (namely, $\mathrm{N}$ is an odd number) have linear $I-V$ curves with the same slope regardless of the ribbon width. For the 55-7 SW N=3 and 5 cases, current has more complicated behavior, firstly the current is increasing in the bias range of 0 to $0.2 \mathrm{~V}$, then shrinks at $0.2 \mathrm{~V}-0.6 \mathrm{~V}$ range and rises in the range of $0.6 \mathrm{~V}$ $1.8 \mathrm{~V}$, before being a conductor again from $1.8 \mathrm{~V}$ bias point.

For a better and intuitive understanding of the $I-V$ characteristics, we study the transmission spectra as functions of the applied bias voltage and electron energy in Fig. 5. As described, the current $I\left(V_{b}\right)$ can be calculated by the Landauer formula which is the integration of the transmission coefficient in the bias window. The two black solid lines in each panel indicate the transport window in which the transmission contributes to the currents. Though the transmission coefficients near the Fermi level decrease at higher bias, the broadened bias window includes more transmission coefficients. This compensates for the loss of electron transport near the Fermi level. There are obvious peaks in transmission spectra at $E_{F}$ under zero bias for both ZGNR N=3 and 55-7 $\mathrm{SW} \mathrm{N}=3$ devices. For ZGNR N=3, the intensity of $T(E)$ is nearly constant until to 0.2 from 2.0 $\mathrm{V}$, which reflects the linear growth of current in Fig. 4. For 55-7 SW N=3 case, the transmission does not decrease until the bias is higher than $0.2 \mathrm{~V}$ so it is reflected in the rise of the current in the 


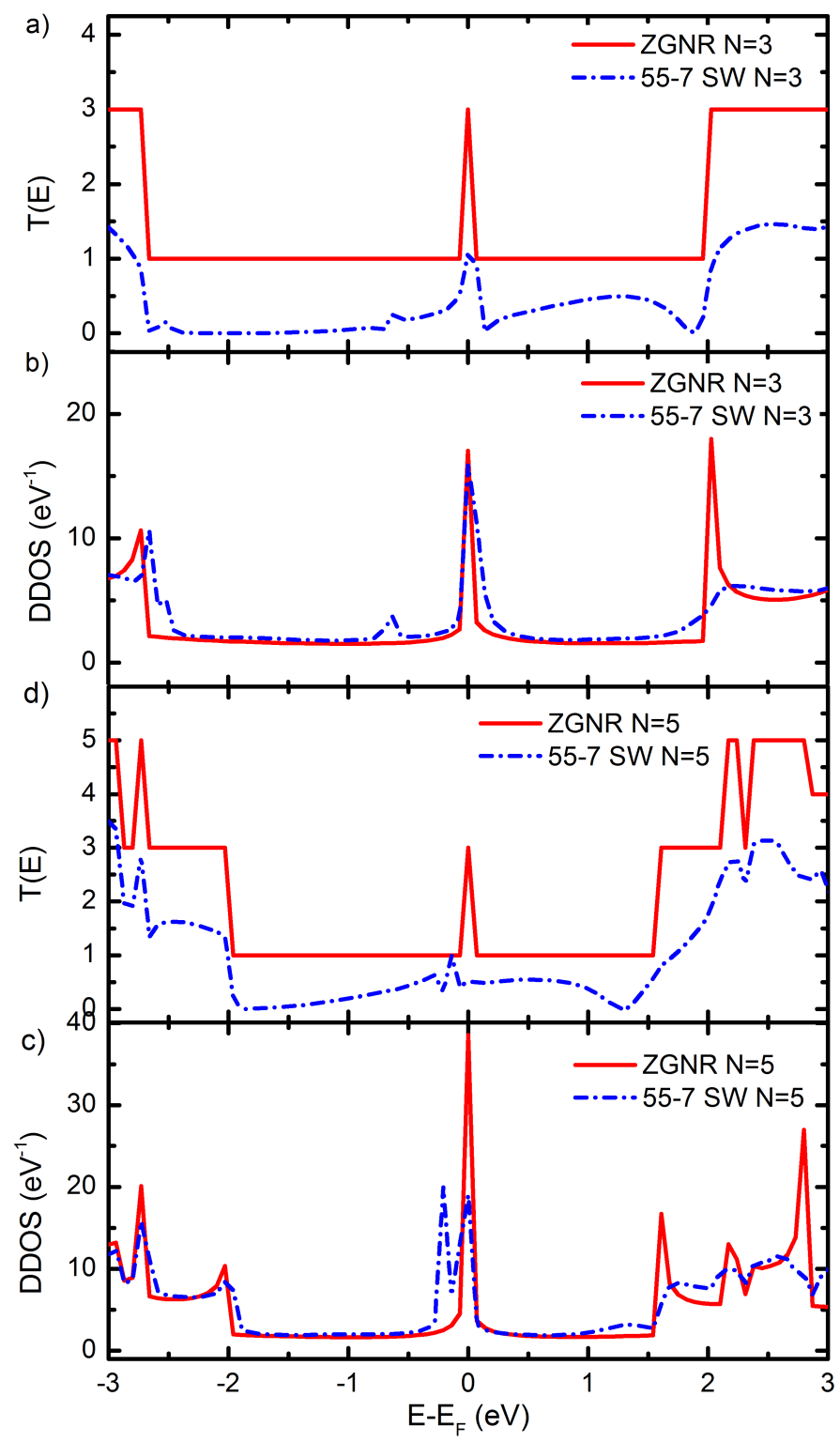

Fig. 3. Transmission spectrum $T(E)(\mathrm{a}, \mathrm{c})$ and Device density of states (DDOS) (b, d) as functions of electron energy at zero bias voltage for ZGNR (solid curves) and 55-7 SW defected (short dash dot curves) devices. Graphics are shown for devices: a), b) for $\mathrm{N}=3$, and c), d) for $\mathrm{N}=5$.

first bias range from 0 to $0.2 \mathrm{~V}$ in Fig. 4. The transmission coefficients keep fast decreasing in the bias region $(0.20 \mathrm{~V}-0.80 \mathrm{~V})$ and then rises again, therefore, we have the same current behavior for these bias ranges. There is an obvious fluctuation with two peaks and two valleys along 55-77 SW $\mathrm{N}=5$, while current increases linearly in the range of $(0.6 \mathrm{~V}, 1.2 \mathrm{~V})$. Since 55-7 SW N=3 and 55-77 
$\mathrm{SW} \mathrm{N}=5$ defect transmission is always weaker than pristine ZGNR N=3 and 5 transmissions in the bias window after, so the SW defect currents through the systems are much smaller than pristine ZGNR currents.

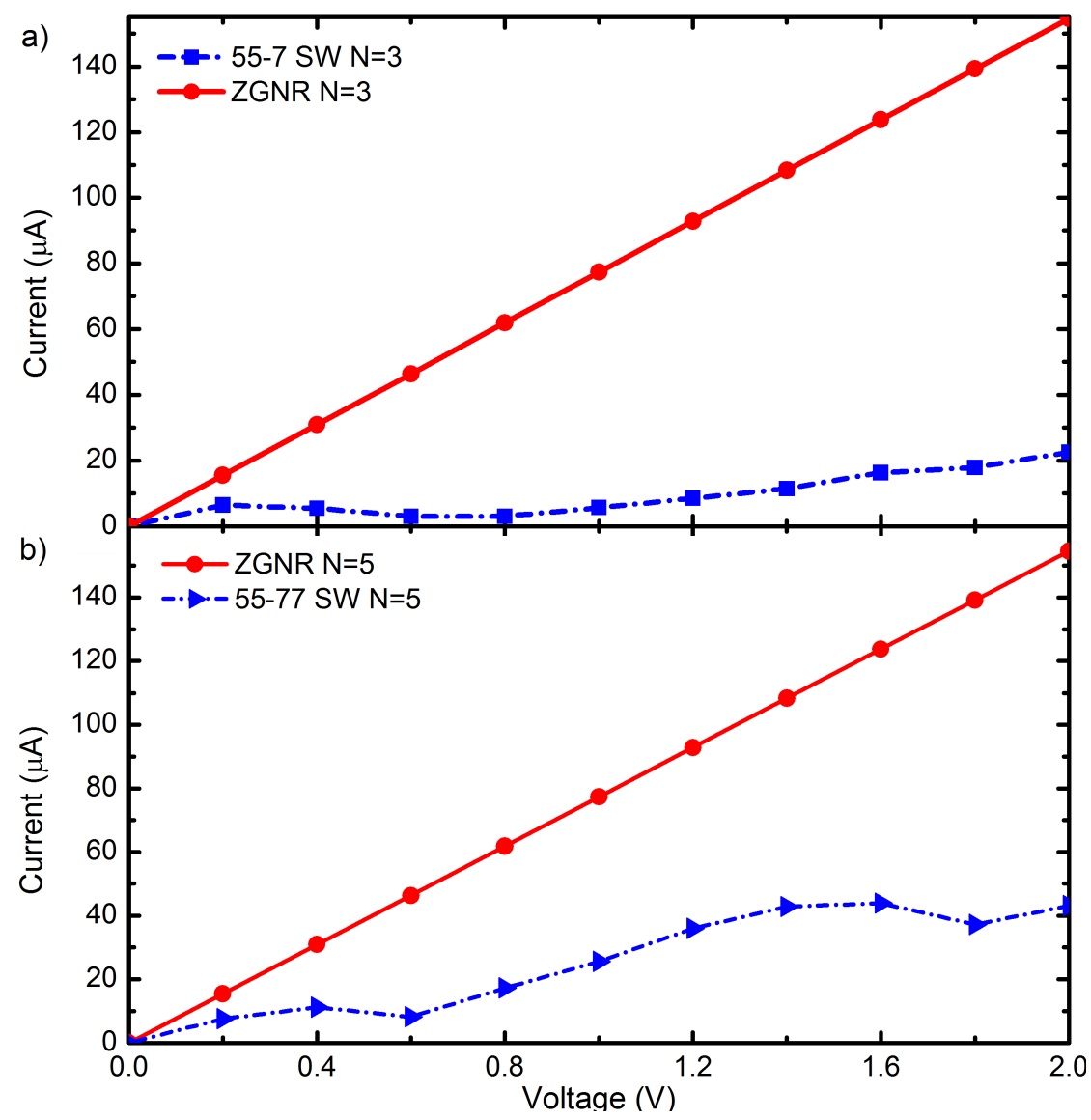

Fig. 4. Calculated $I-V$ curves for the structures: a) ZGNR N=3 (solid curves) and 55-7 $\mathrm{SW} N=3$ (short dash dot curves); b) ZGNR N=5 (solid curves) and 55-7 SW N=5 (short dash dot curves).

For the transmission spectrum of ZGNR N=3, 55-7 SW N=3 and ZGNR N=5, 55-77 SW $\mathrm{N}=5$ cases at increasing bias (see Figs. 6a-6d) from zero to $1.8 \mathrm{~V}$. The value of $T(E)$ for ZGNR $\mathrm{N}=3$ and 5 devices remain unchanged at Fermi energy, while for the transmission spectrum of ZGNR N=5, there are perfect transmission steps. One more interesting thing is that there exists unitized transmission flats around the Fermi level instead of the Fermi peak of ZGNR, which indicates the ballistic transport. However, the electronic transmissions are small within the bias window under low bias in 55-7 SW N=3 and 55-77 SW N=5 devices, corresponding to the small currents. As the bias is increased up to about $1.2 \mathrm{~V}$, obvious transmission peaks begin to enter the bias window, that is, some transmission channels begin to contribute to the current. 

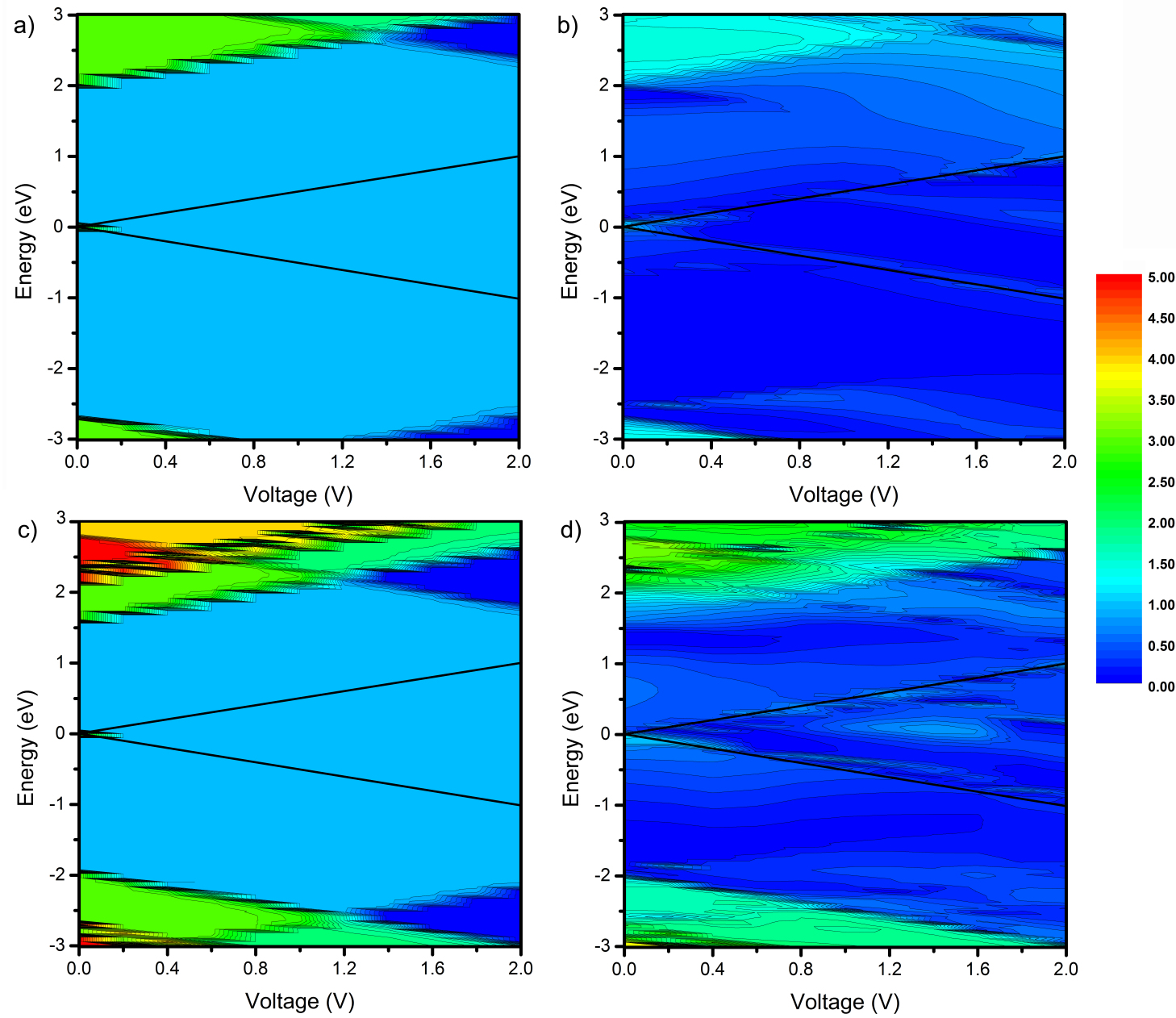

Fig. 5. Contours of the bias-dependent transmission $T\left(E, V_{b}\right)$ of the structures: (a) ZGNR $\mathrm{N}=3$; (b) 55-7 SW N=3; c) ZGNR N=5 and d) 55-77 SW N=5.

We have studied the local density of states (LDOS) of the devices at the Fermi level under zero bias voltage. The LDOS of GNR N=3 device (Fig. 7a) is mainly located at $\mathrm{C}$ atoms on the two edges of ribbon, thus the transmission platform appears in Fig. 3a and the electric transport has a metallic character. For 55-7 SW N=3 device, LDOS becomes smaller at the edge of the defect than that at the other sites (see Fig. 7b). Moreover, LDOS is not uniformly located at $\mathrm{C}$ atoms on two edges of ribbon, thus explains the transmission decrease appearing in Fig. 3b. Comparing Fig. 7d (for 55-77 SW N=5) and Fig. 7c (for ZGNR N=5), it has been clear that the DOS is nonlocal along the up edge of the device, but is suppressed at the defect along the down edge, thus the corresponding transmission peak decreases compared with that of the pristine ZGNR N=5 device. 

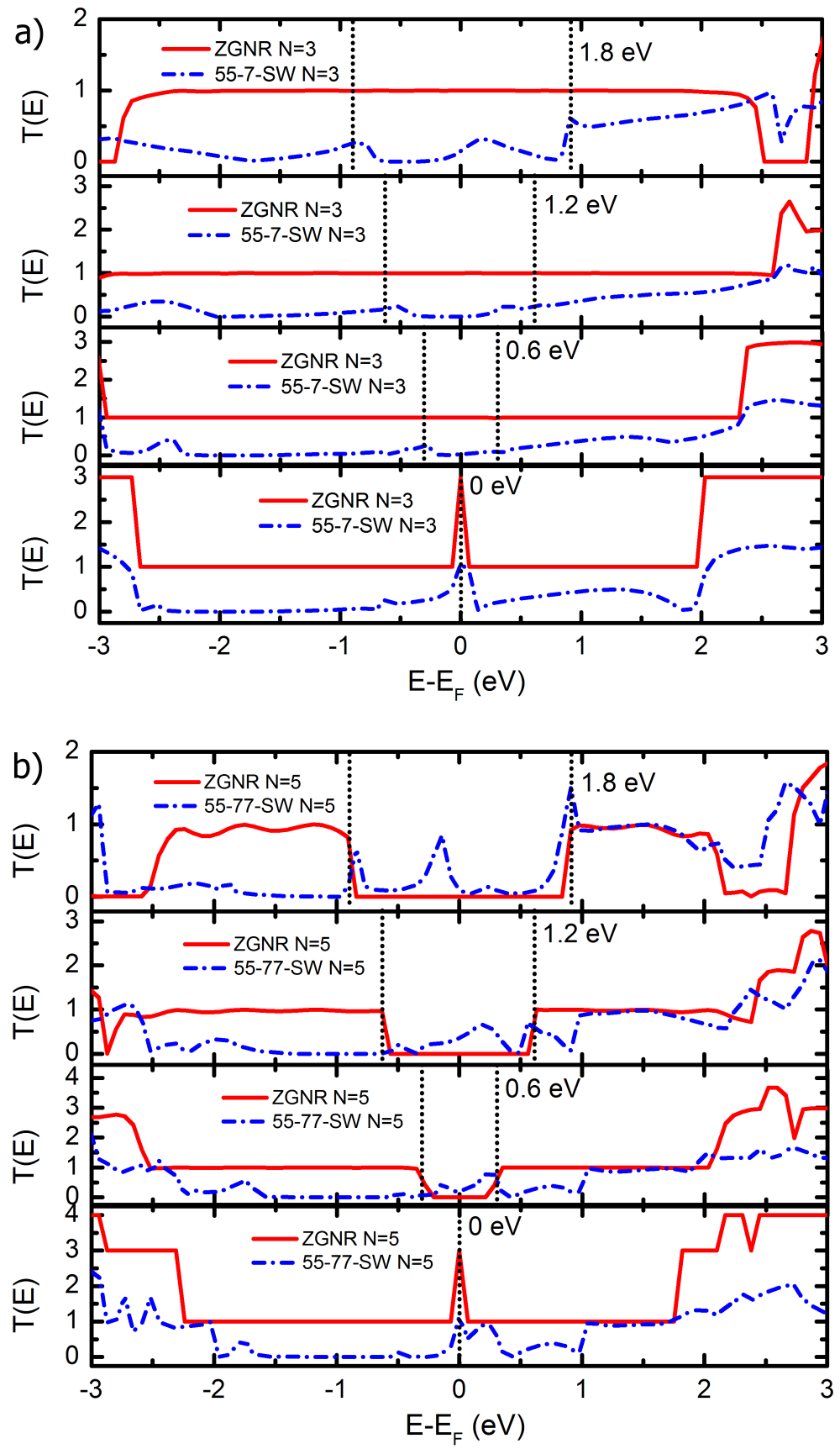

Fig. 6. Transmission spectra of the ZGNR $N=3$ and 55-7 $\mathrm{SW} \mathrm{N}=3$ (a), ZGNR $\mathrm{N}=5$ and 55-77 SW N=5 (b) devices at different bias voltages $(\triangle \mathrm{V}=0.0 \mathrm{~V}, 0.6 \mathrm{~V}, 1.2 \mathrm{~V}$ and $1.8 \mathrm{~V})$. The vertical dashed lines represent the bias windows. 


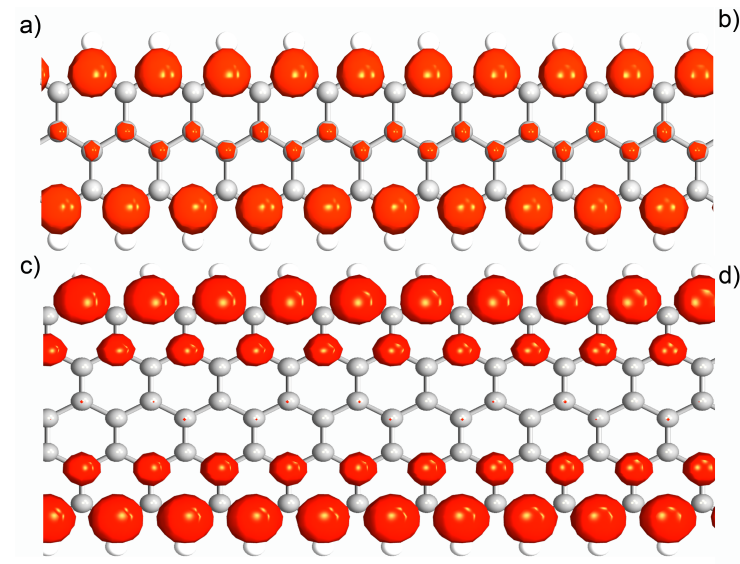

b)

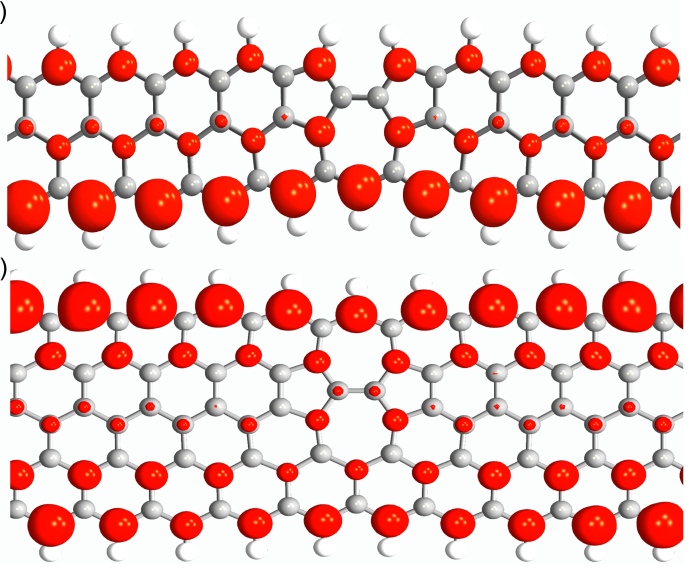

Fig. 7. Spatial distribution of LDOS at zero bias for the: a) ZGNR N=3; b) 55-7 SW $\mathrm{N}=3, \mathrm{c}) \mathrm{ZGNR} \mathrm{N}=5$ and d) 55-77 $\mathrm{SW} \mathrm{N}=5$.

\section{The 55-77 SW N=4 and 6 defects}

We present the transmission spectra $T(E)$ and device density of states (DDOS) at zero voltage for the cases $\mathrm{N}=4$ (Figs. 8a,b) and 6 (Figs. 8c,d), i.e. for the ones of the symmetrical SW defects. In Fig. 8a and 8c the zero bias transmission spectra, $T(E)$ (solid curves), as a function of the electronic energy in the defect-free ZGNRs, exhibit a sequence of steps of integer transmission and an enhanced transmission at the Fermi level, which are typical for graphene nanoribbons. In the case of 55-77 SW N=4 and 6 defective ZGNRs (short dash dot curvess in Figs. 8a and 8c), the conductance peak, as a result of the four $\mathrm{H}$ atom shrinking [21], in the vicinity of charge neutrality point (CNP), is decreased approximately a half. When a SW defect on the ZGNR, the transmission spectrum changes a lot, and one can find that the transmission steps are destroyed, instead, so the transmission dips appear. The conductance with symmetric defects remarkably decreases below the CNP, manifesting monotonous reduction of conductance with increasing electron energy. A complete electron backscattering arising from the SW defect induced quasibound states is located approximately at $1.68 \mathrm{eV}$ for case of $\mathrm{N}=4$ and nearly at $1.12 \mathrm{~V}$ for $\mathrm{N}=6$. In addition, in the $\mathrm{SW}$ defect configuration, symmetrical effects yield a remarkable conductance decrease characterized by a long tail below the CNP, and eventually the lowest conductance occurring at $-2.24 \mathrm{eV}(\mathrm{N}=4)$ or $-1.72 \mathrm{~V}(\mathrm{~N}=6)$, suggesting the electronic transport is almost fully suppressed. The striking device density of states peaks in Fig. 8b of the 55-77 SW N=4 defect induced by the edge states are remarkably decreased compared to ZGNR N=4. It can be seen that one small peak appears in the 55-77 SW N=6 DDOS, corresponding to the energies of $0.12 \mathrm{eV}$. The transmission dips of 55-77 SW N=6 appear just right in the energies of $1.12 \mathrm{eV}$, which is the same as that of the one DDOS states peak that appear. As a consequence, the conductance of 55-77 SW N=4 and 6 defect configurations are decreased substantially in the vicinity of CNP, and such a decrease indicates that SW defect site significantly lowers the edge state.

The transmission spectrum of ZGNR N=4 and 55-77 SW N=4 (see Fig. 9a), as well as of ZGNR N=6 and 55-77 SW N=6 (Fig. 9b) have been presented for the increasing bias from 0V to $1.8 \mathrm{~V}$. Numerical results show that for pristine $\mathrm{ZGNR} \mathrm{N}=4 T(E)$ almost remains unchanged by 


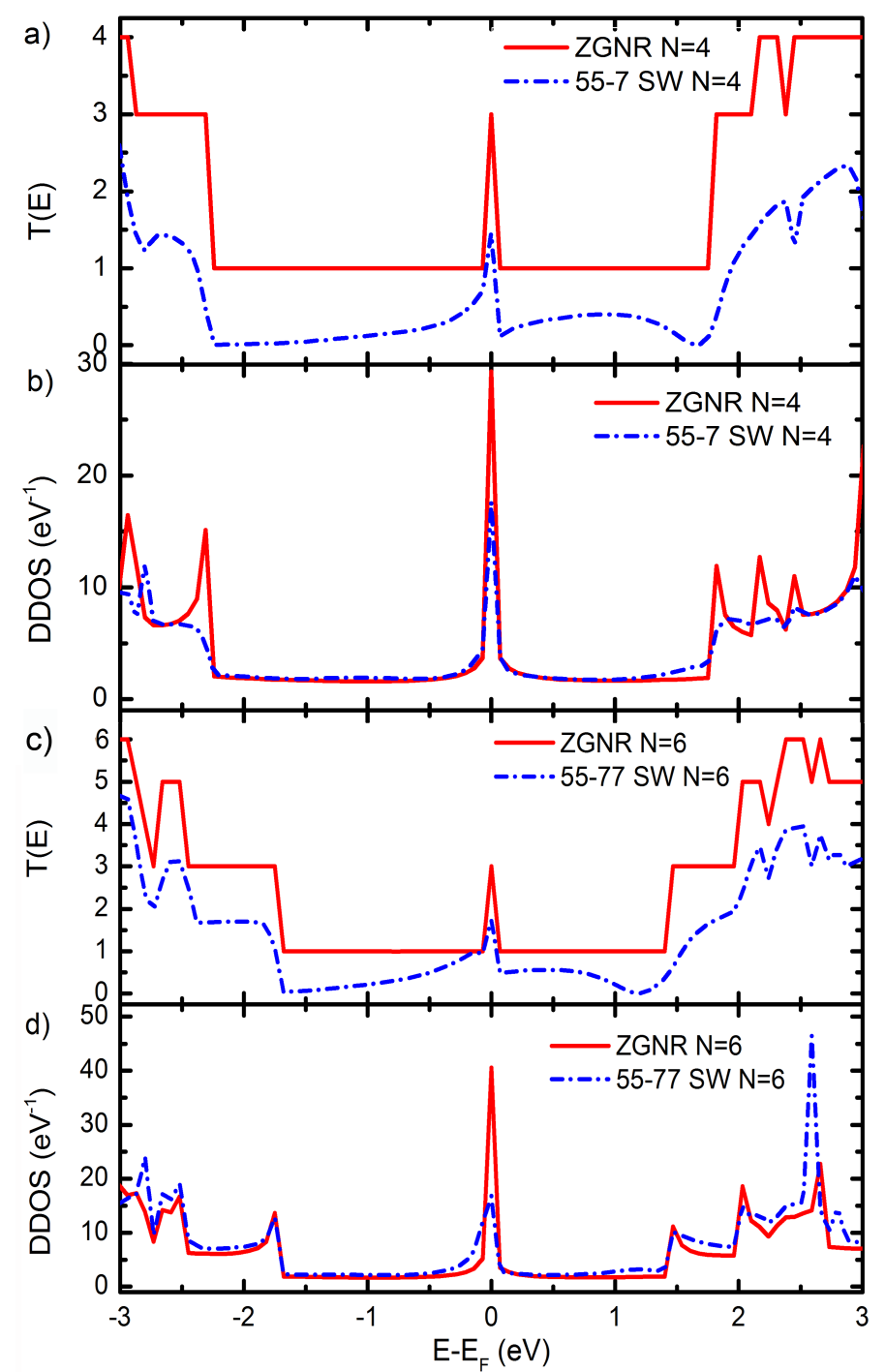

Fig. 8. Transmission spectrum $T(E)$ (a,c) and Device density of states (DDOS) (b, d) as functions of electron energy at zero bias voltage for the ZGNR (solid curves) and 55-7 SW defected (short dash dot curves) devices. Graphics are shown for the devices: a), b) for $\mathrm{N}=4$, and c), d) for $\mathrm{N}=6$.

the increasing of bias voltage. While 55-77 SW N=4 defect case the width of transmission flat around the Fermi level increases when the bias increases until $2.0 \mathrm{~V}$, whereas the intensity remains nearly unaffected. Dealing with the ZGNR N=6 and 55-77 SW N=6 defective devices, there are transmission coefficients in bias windows, resulting in the current increase rapidly with the applied bias voltage at lower bias region. For 55-77 SW N=6 defect case, there is a smaller transmission coefficient in low bias window, so the current is smaller than ZGNR $\mathrm{N}=6$ pristine. The energy gap appears and becomes more widened with increasing bias in ZGNRs, which contributes to the 
transformation from half-metallicity to semiconductor. As seen from Fig. 9 for both the widtnesses $\mathrm{N}=4$ and 6, except the effect of shrinking four $\mathrm{H}$ atoms at zero bias, the transmission coefficients for the SW defective ZGNRs, as well as for the pristine ones, critically fall down, that explains the very small current values.
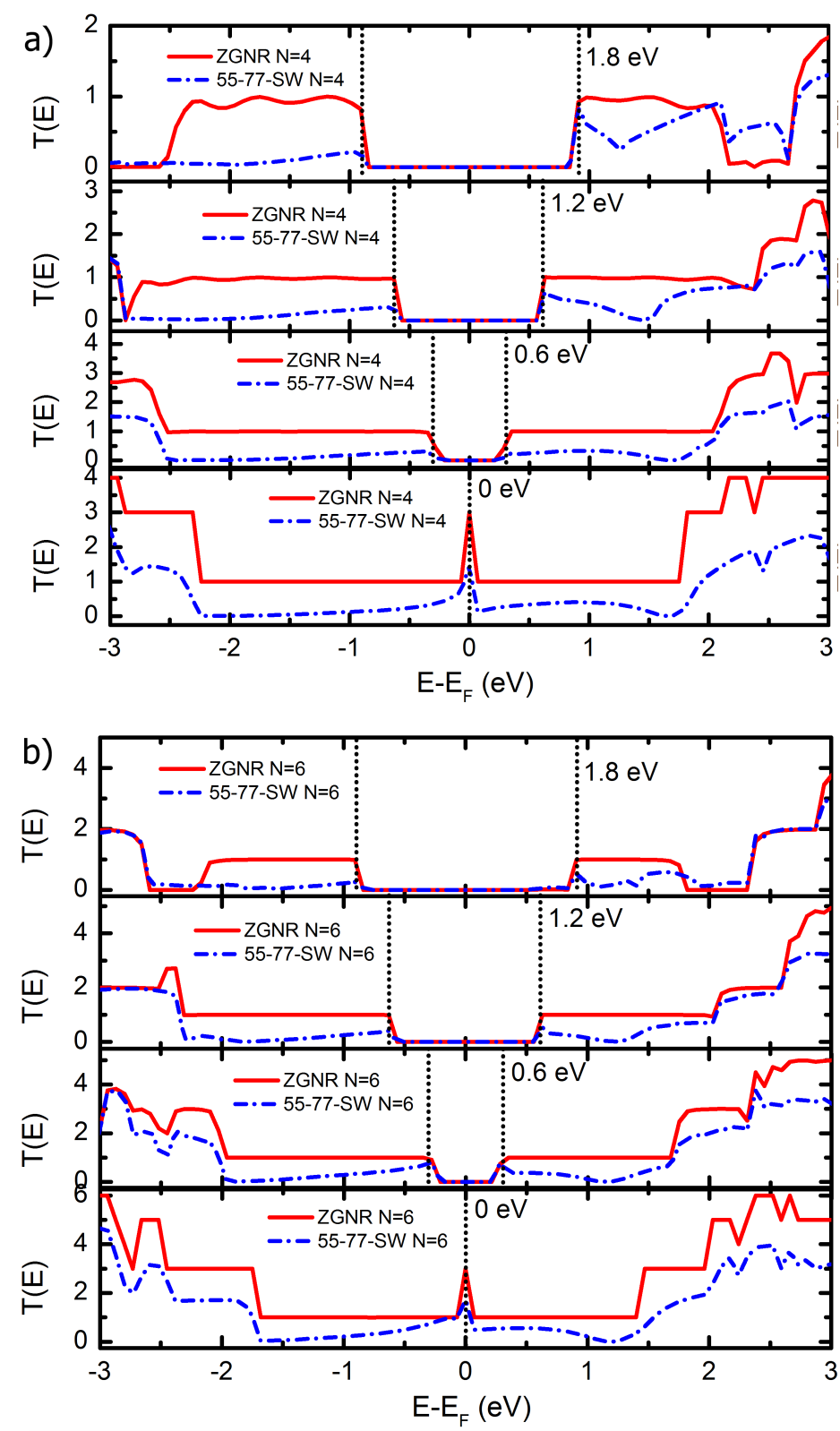

Fig. 9. Transmission spectrum of the ZGNR $N=4$ and 55-77 $\mathrm{SW} N=4$ (a), ZGNR $N=6$ and 55-77 SW N=6 (b) devices at different bias voltages. The vertical dashed lines represent the bias windows. 
For the case of symmetric ZGNRs, the study of the transmission spectra as a function of the applied bias voltage and electron energy has been performed. Similar to that shown in [13], for the cases of asymmetric ZGNRs (see Fig. 4 and 5) there are fairly big transmission coefficients, leading to an increase in current. However, for the symmetric ZGNRs with and without SW single defect presented in Fig. 10, there is the fact that transmission coefficients with a narrow energy range within bias windows, causing a smaller current in comparison of the one for asymmetric ZGNRs. For the transmission spectrum of ZGNR $\mathrm{N}=6$ device, there are perfect transmission steps. When a SW defect on the ZGNR, the transmission spectrum changes a lot, and one can find that the transmission steps are destroyed, instead one transmission dips appear at $1.12 \mathrm{eV}$ energy sites, respectively.
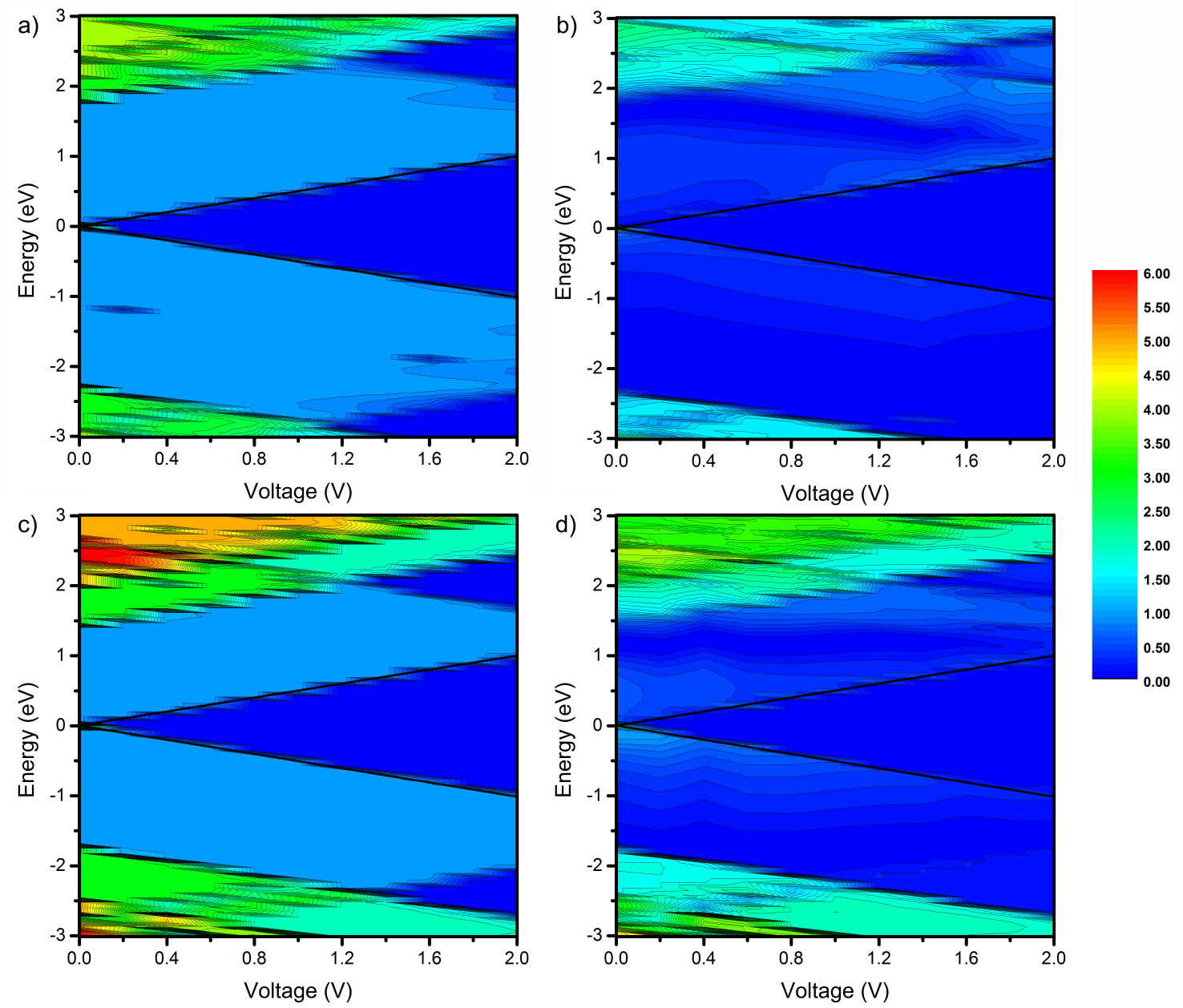

Fig. 10. Contour of the bias-dependent transmission $\mathrm{T}\left(\mathrm{E}, V_{b}\right)$ of the structures: a) ZGNR $\mathrm{N}=4$, b) 55-7 SW N=4, c) ZGNR N=6 and d) 55-7 SW N=6.

We next turn to study the current-voltage ( $I-V)$ curves as a function of bias (see Fig. 11). The current values of 55-77 SW N=4 device are dramatically small in comparison with those of 
ZGNR N=4 one (Fig. 11a). There is an obvious fluctuation with four peaks and three valleys along ZGNR N=4 and 55-77 SW N=4 defects. The current is increased in $(0-0.4 \mathrm{~V}),(0.8 \mathrm{~V}-1.0 \mathrm{~V})$, $(1.2 \mathrm{~V}-1.4 \mathrm{~V})$ and holds out the peaks at $1.0 \mathrm{~V}$ and $1.4 \mathrm{~V}$. When the bias is larger than $1 \mathrm{~V}$ and $1.4 \mathrm{~V}$, the current depressions are observed in ranges $(1.0 \mathrm{~V}-1.2 \mathrm{~V})$ and $(1.4 \mathrm{~V}-1.6 \mathrm{~V})$, indicating an obvious NDR behavior. For the pristine ZGNR N=6, the current is close to zero under finite bias and begins to increase when the bias is stronger than a threshold bias voltage. Comparing the I-V curves of 55-77 SW N=6 device with its counterpart (Fig. 11b), it can be established the occurrence of NDR and the current of ZGNR N=6 is larger. Therefore, both of the pristine and SW-defective symmetric ZGNRs are semiconductor [22] and possess a dual functionality, which is important to further improve the level of integration of future atomic-scale circuits.

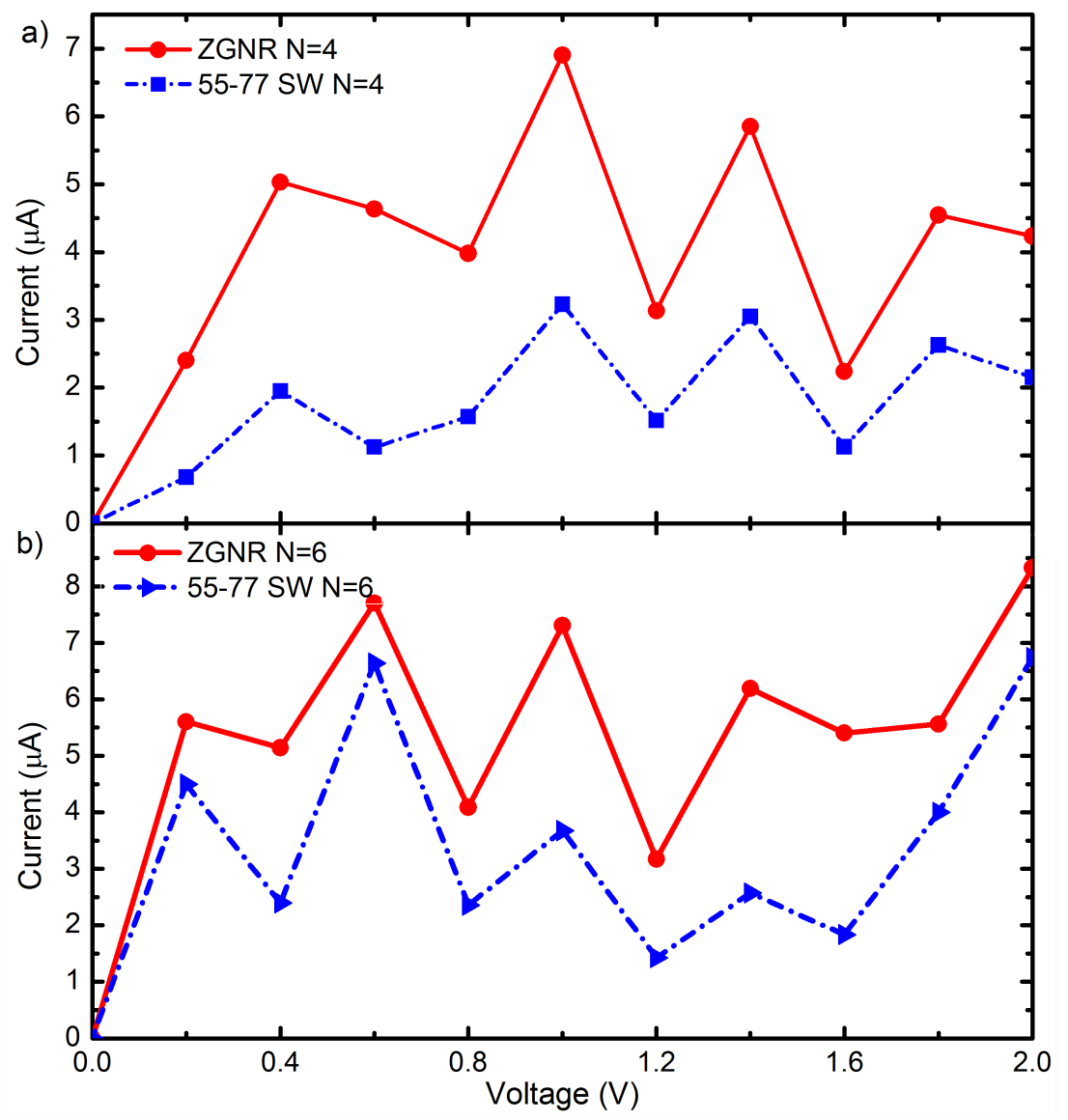

Fig. 11. Calculated $I-V$ curves for the structures: a) $Z G N R ~ N=4$ and $55-77 \mathrm{SW} \mathrm{N}=4$; b) ZGNR N=6 and 55-77 SW N=6.

In order to further enlighten the semiconductor behavior of the symmetric ZGNRs' quantum transport, we have calculated the corresponding LDOS, as shown in Fig. 12. For the pristine ZGNR structures, it can be seen that the density of states is almost localized at the $\mathrm{C}$ atoms on the 
edges of asymmetric ZGNR (see Fig. 7c for ZGNR N=5), whereas LDOS distribution is delocalized in the whole scattering regions for ZGNRs with $\mathrm{N}=4$ and 6 (see Figs. 12a and 12c). The level of the delocalization decreases with the width of symmetric ZGNRs. It has been attributed [21] that these distinct transport behaviors in asymmetric and symmetric ZGNRs to the different coupling between the conducting subbands influenced by the wave function modification around the CNP. The presence of the only SW defect in the scattering region remarkably enhances the LDOS delocalization overall $\mathrm{C}$ chains in the scattering region in Figs. $12 \mathrm{~b}$ and $12 \mathrm{~d}$, that explains the obvious decreasement of currents in the defective devices referred to those in the pristine counterparts. However, since the single SW defect occurrence here enforces only backscattering events, but can not cause a qualitative change of the relative LDOS redistribution picture between the edges and the scattering region in the devices, so the current-voltage curve for the defective ZGNR follows the semiconductor behavior of that with NDR for the pristine counterpart.

The dips of current in Fig. 11 can be caused by strong electron backscattering induced by the coupling between all states are expanded to lead to full suppression of the conduction channel at particular resonance energies [21]. More details, the origin of rises and NDR in the $I-V$ characteristics is eplained by the changing numbers of the frontier molecular orbitals, entering to the bias windows $[12,13]$. If the orbital is delocalized across the molecule, an electron that enters the molecule at the energy of the orbital has a high probability of going through the molecule. For pristine symmetric ZGNRs the current increases even at very small bias, some molecular orbitals, such as highest occupied molecular orbital (HOMO), first lowest unoccupied molecular orbitals (LUMO), etc., can be localized in bias windows. The occurrence of SW defect into ZGNR leads to a separation between spin-up and spin-down molecular orbitals, so it may alter the number of molecular orbitals entering in bias windows or outgoing from there.

a)

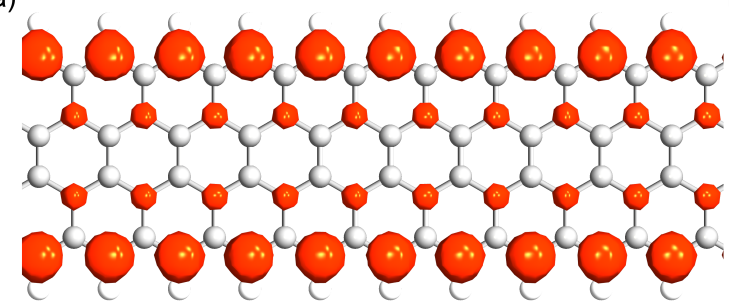

c)

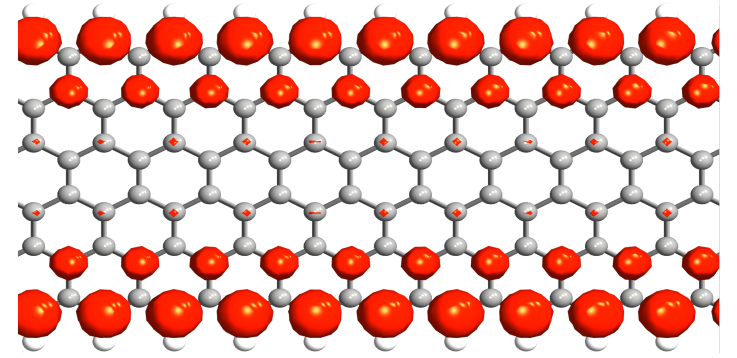

b)

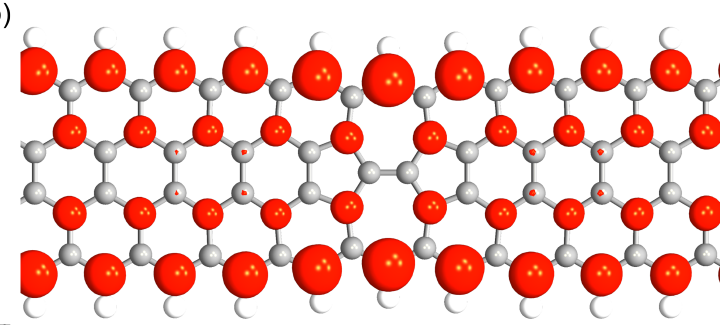

d)

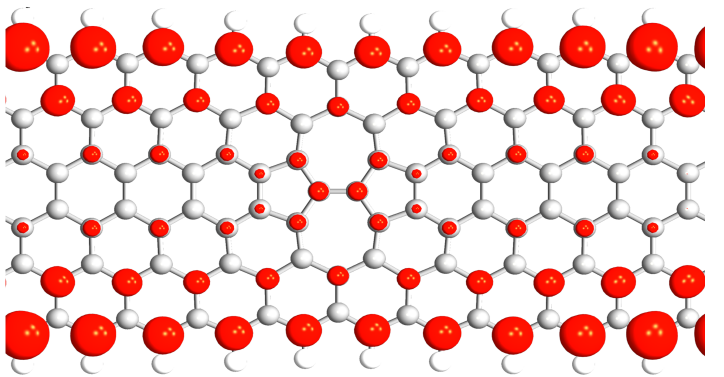

Fig. 12. Spatial distribution of LDOS for the a) ZGNR N=4, b) 55-77 SW N=4, c) ZGNR $\mathrm{N}=6$, and d) 55-77 SW N=6 structures, respectively, corresponding to the energy windows bias zero. 


\section{CONCLUSIONS}

In summary, we have studied the formation of SW defect in ZGNRs with various widthness and the defect influences on electronic transport properties of both the asymmetric and symmetric ZGNRs, using the non-equilibrium Green's function (NEGF) technique, combined with the density functional theory.Our results confirm the metallicity in the conductance of the asymmetric ZGNRs, meanwhile for the symmetric ZGNRs the behavior of electronic transport is more complex - the $I-V$ curves obey a semiconductor character, with NDR. The SW defect situating in both types (asymmetric/symmetric) of the ZGNRs significantly depresses the currents passing through $\mathrm{C}$ chains in the scattering regions, but does not change the character of their electronic transport.

\section{ACKNOWLEDGMENTS}

This research is funded by Vietnam National Foundation for Science and Technology Development (NAFOSTED) under grant number 103.01-2017.74. Also, we would like to thank the Swedish National Infrastructure for Computing (SNIC) for the computational support.

\section{REFERENCES}

[1] K. S. Novoselov, A. K. Geim, S. V. Morozov, D. Jiang, Y. Zhang, S. V. Dubonos, I. V. Grigorieva and A. A. Firsov, Science 306 (2004) 666.

[2] K. S. Novoselov, A. K. Geim, S. V. Morozov, D. Jiang, M. I. Katsnelson, I. V. Grigorieva, S. V. Dubonos and A. A. Firsov, Nature 438 (2005) 197-.

[3] J. P. Llinas, A. Fairbrother, G. B. Barin, W. Shi, K. Lee, S. Wu, B. Y. Choi, R. Braganza, J. Lear, N. Kau, W. Choi, C. Chen, Z. Pedramrazi, T. Dumslaff, A. Narita, X. Feng, K. Müllen, F. Fischer, A. Zettl, P. Ruffieux, E. Yablonovitch, M. Crommie, R. Fasel and J. Bokor, Nature Communications 8 (2017) 633.

[4] M. Choudhury, Y. Yoon, J. Guo and K. Mohanram, Proceedings of the 45th annual Design Automation Conference (2008) 272.

[5] F. Banhart, J. Kotakoski and A. V. Krasheninnikov, Acs Nano 5 (2011) 26.

[6] J. Lahiri, Y. Lin, P. Bozkurt, I. I. Oleynik and M. Batzill, Nature Nanotechnology 5 (2010) 326.

[7] G. D. Lee, C. Z. Wang, E. Yoon, N. M. Hwang, D. Y. Kim and K. M. Ho, Physical Review Letters 95 (2005) 205501.1.

[8] L. Li, S. Reich and J. Robertson, Physical Review B 72 (2005) 184109.1.

[9] Z. Li, B. Huang and W. Duan, Journal of Nanoscience and Nanotechnology 10 (2010) 5374-.

[10] X. Li, D. Zou, B. Cui, C. Fang, J. Zhao, D. Li and D. Liu, The Royal Society of Chemistry 20177 (2017) $25244-$.

[11] K. Li and X.-H. Zhang, Physics Letters A 382 (2018) 1167.

[12] W. Liu, F.-H. Meng, J.-H. Zhao and X.-H. Jiang, Theoretical and Computational Chemistry 16 (2017) 1750032.1.

[13] Y. Ren and K.-Q. Chen, Applied physics 107 (2010) 044514.1.

[14] J. P. Perdew, K. Burke and M. Ernzerhof, Physical Review Letters 77 (1996) 3865.

[15] H. J. Monkhorst and J. D. Pack, Physical Review B 13 (1976) 5188.

[16] S. Grimme, Journal of Computational Chemistry 27 (2006) 1787.

[17] R. Landauer, IBM Journal of Research and Development 1 (1957) 223.

[18] S. Datta, Electronic transport in mesoscopic systems, Cambridge university press, 1997.

[19] J. Ma, D. Alfè, A. Michaelides and E. Wang, Physical Review B 80 (2009) 033407.1.

[20] R. Dettori, E. Cadelano and L. Colombo, Journal Of Physics: Condensed Matter 24 (2012) 104020.1.

[21] H. Zeng, J.-P. Leburton, Y. Xu and J. Wei, Nanoscale Research Letters 6 (2011) 254.1.

[22] Y. Zou, M. Long, M. Li, X. Zhang, Q. Zhang and H. Xu, The Royal Society of Chemistry 5 (2015) 19152-. 